\title{
Good Geometry on the Curve Moduli
}

\author{
Dedicated to Heisuke Hironaka
}

By

Kefeng LiU*, Xiaofeng SuN** and Shing-Tung YAU***

\section{Contents}

$\S 1 . \quad$ Introduction

$\S 2$. Background and Notation

$\S 3$. Goodness of the Weil-Petersson Metric

$\S 4$. Dual Nakano Negativity of the Weil-Petersson Metric

$\S 5 . \quad L^{2}-$ Cohomology and Rigidity

$\S 6$. Goodness of the Ricci and Perturbed Ricci Metrics

References

\section{$\S 1$. Introduction}

This paper is written to dedicate to Professor Hironaka for his outstanding contributions to mathematics, especially in algebraic geometry. His leadership in Asia has inspired many generations of asian mathematicians. We wish many young mathematicians will continue to follow his footsteps in this grand subject.

In this paper we describe some of our recent results in the asymptotic analysis of various Kähler metrics and their curvatures on the moduli spaces of Riemann surfaces. These works will enable us to use differential geometric techniques to study various algebraic geometric and topological problems about

Communicated by K. Saito. Received January 30, 2007.

2000 Mathematics Subject Classification(s): 14D20, 14H20, 32G13, 53C55.

The authors are supported by NSF grants.

* Center of Mathematical Sciences, Zhejiang University, Hangzhou, China; Department of

Mathematics, University of California at Los Angeles.

** Department of Mathematics, Lehigh University.

*** Center of Mathematical Sciences, Zhejiang University, Hangzhou, China; Department of

Mathematics, Harvard University.

(c) 2008 Research Institute for Mathematical Sciences, Kyoto University. All rights reserved. 
the moduli spaces. We believe that the new results here may give a new way to study the intersection theory on various moduli spaces.

In his work [11], Mumford defined the goodness condition to study the currents of Chern forms defined by a singular Hermitian metric on a holomorphic bundle over a quasi-projective manifold. The goodness condition is a growth condition of the Hermitian metric near the compactification divisor of the base manifold. The major property of a good metric is that the currents of its Chern forms define the Chern classes of this bundle. For details, please see Section 2.

Except for the symmetric spaces discussed by Mumford in [11], several natural bundles over moduli spaces of Riemann surfaces give beautiful and useful examples. In [20], Wolpert showed that the metric induced by the hyperbolic metric on the twisted relative tangent bundle over the total space of moduli space of hyperbolic Riemann surfaces is good. Later it was shown by Trapani [16] that the metric induced by the Weil-Petersson metric on the determinant line bundle of the logarithmic cotangent bundle of the Deligne-Mumford moduli space is good. In both cases, the bundles involved are line bundles in which cases it is easier to estimate the connection and curvature. Other than these, very few examples of natural good metrics are known.

The goodness of the Weil-Petersson metric has been a long standing open problem. In this paper, we describe our solution of this problem. In fact we will present proofs of the goodness of the metrics induced by the Weil-Petersson metric, as well as the Ricci and perturbed Ricci metrics on the logarithmic cotangent bundle over the compactified moduli space of Riemann surfaces (the DM moduli spaces). These works depend on our very accurate estimates of the asymptotic of the curvature and connection forms of these metrics in [6] and [7] together with the estimates of derivatives of the hyperbolic metric on Riemann surfaces. The computations and proofs are quite involved and very subtle. We will also present our proof of the dual Nakano negativity of the Weil-Petersson metric. In [13] Schumacher proved the strong negativity of the Weil-Petersson metric in the sense of Siu. Dual Nakano negativity is stronger than the strong negativity in the sense of Siu and several interesting consequences will follow. For example the goodness, combining with the dual Nakano negativity of the Weil-Petersson metric, gives rise to interesting geometric consequences such as infinitesimal rigidity of the complex structure on the moduli spaces. We feel that our results open a new way to study the intersection theory on the moduli spaces by using differential geometric techniques, and also to apply index theory to the study of the geometry and topology of the moduli spaces and Teichmüller spaces. 
Now we briefly describe the organization of this note. In Section 2 we recall some known works on the geometry of moduli spaces which include the degeneration of Riemann surfaces and hyperbolic metrics, the Ricci, perturbed Ricci and Kähler-Einstein metrics as well as their curvature properties, the asymptotic of Weil-Petersson metric, the Ricci and perturbed Ricci metrics as established in [6] and [7]. We also review Mumford's definition of good metrics.

In Section 3 we describe the main ideas of proving the goodness of the WeilPetersson metric and in Section 4 we describe the proof of the dual Nakano negativity of the Weil-Petersson metric. In Section 5 we apply these results to derive the vanishing theorem about certain cohomology groups and the infinitesimal rigidity of the moduli spaces. Our definition of the $L^{2}$ cohomology generalizes the usual one with trivial bundle coefficients. Here we have used both the Weil-Petersson metric and the Ricci metric and their goodness.

Finally in Section 6 we present the goodness of the Ricci and the perturbed Ricci metrics. We remark that our previous results about the asymptotic behavior of the Kähler-Einstein metrics on the moduli spaces already imply an orbifold Chern number inequality for the logarithmic cotangent bundle which should give new information about positive divisors on the DM moduli spaces. In this paper, we will only give the main ideas and sketch the proofs of the results presented. For details and precise estimates, we refer the reader to [8] and $[9]$.

We would like to thank professors H.-D. Cao, R. Schoen and E. Viehweg for their help and encouragement.

\section{§2. Background and Notation}

In this section we review the necessary backgrounds and setup our notations. Most of the results can be found in [6], [7], [20] and [11].

Let $\mathcal{M}_{g, k}$ be the moduli space of Riemann surfaces of genus $g$ with $k$ punctures such that $2 g-2+k>0$. We know there is a unique hyperbolic metric on such a Riemann surface. To simplify the computation, throughout this paper, we will assume $k=0$ and $g \geq 2$ and work on $\mathcal{M}_{g}$. Most of the results can be trivially generalized to $\mathcal{M}_{g, k}$.

By the Riemann-Roch theorem, we know that the complex dimension of the moduli space is $n=\operatorname{dim}_{\mathbb{C}} \mathcal{M}_{g}=3 g-3$. Given a Riemann surface $X$ of genus $g \geq 2$, we denote by $\lambda$ the unique hyperbolic (Kähler-Einstein) metric on $X$. Let $z$ be local holomorphic coordinate on $X$. We normalize $\lambda$ :

$$
\partial_{z} \partial_{\bar{z}} \log \lambda=\lambda
$$


Let $\left(s_{1}, \cdots, s_{n}\right)$ be local holomorphic coordinates on $\mathcal{M}_{g}$ near a point $p$ and let $X_{s}$ be the corresponding Riemann surfaces. Let $\rho: T_{s} \mathcal{M}_{g} \rightarrow$ $H^{1}\left(X_{s}, T X_{s}\right) \cong H^{0,1}\left(X_{s}, T X_{s}\right)$ be the Kodaira-Spencer map. Then the harmonic representative of $\rho\left(\frac{\partial}{\partial s_{i}}\right)$ is given by

$$
\rho\left(\frac{\partial}{\partial s_{i}}\right)=\partial_{\bar{z}}\left(-\lambda^{-1} \partial_{s_{i}} \partial_{\bar{z}} \log \lambda\right) \frac{\partial}{\partial z} \otimes d \bar{z}=B_{i}
$$

If we let $a_{i}=-\lambda^{-1} \partial_{s_{i}} \partial_{\bar{z}} \log \lambda$ and let $A_{i}=\partial_{\bar{z}} a_{i}$, then the harmonic lift $v_{i}$ of $\frac{\partial}{\partial s_{i}}$ is given by

$$
v_{i}=\frac{\partial}{\partial s_{i}}+a_{i} \frac{\partial}{\partial z}
$$

The well-known Weil-Petersson metric $\omega_{W P}=\frac{\sqrt{-1}}{2} h_{i \bar{j}} d s_{i} \wedge d \bar{s}_{j}$ on $\mathcal{M}_{g}$ is defined to be

$$
h_{i \bar{j}}(s)=\int_{X_{s}} A_{i} \bar{A}_{j} d v
$$

where $d v=\frac{\sqrt{-1}}{2} \lambda d z \wedge d \bar{z}$ is the volume form on $X_{s}$. It was proved by Ahlfors that the Ricci curvature of the Weil-Petersson metric is negative. The upper bound of the Ricci curvature of the Weil-Petersson metric was conjectured by Royden and was proved by Wolpert [18].

In our work [6] we defined the Ricci metric $\omega_{\tau}$ :

$$
\omega_{\tau}=-\operatorname{Ric}\left(\omega_{W P}\right)
$$

and the perturbed Ricci metric $\omega_{\widetilde{\tau}}$ :

$$
\omega_{\widetilde{\tau}}=\omega_{\tau}+C \omega_{W P}
$$

where $C$ is a positive constant. These new Kähler metrics have good curvature and asymptotic properties and play important roles in our study.

Now we describe the curvature formulae of these metrics. Please see [6] and [7] for details. We denote by $f_{i \bar{j}}=A_{i} \bar{A}_{j}$ where each $A_{i}$ is the harmonic Beltrami differential corresponding to the local holomorphic vector field $\frac{\partial}{\partial s_{i}}$. It is clear that $f_{i \bar{j}}$ is a function on $X$. We let $\square=-\partial_{z} \partial_{\bar{z}}$ be the Laplace operator, let $T=(\square+1)^{-1}$ be the Green operator and let $e_{i \bar{j}}=T\left(f_{i \bar{j}}\right)$. The functions $e_{i \bar{j}}$ and $f_{i \bar{j}}$ are building blocks of these curvature formulae.

The curvature formula of the Weil-Petersson metric was given by

$$
R_{i \bar{j} k \bar{l}}=-\int_{X_{s}}\left(e_{i \bar{j}} f_{k \bar{l}}+e_{i \bar{l}} f_{k \bar{j}}\right) d v
$$


This formula was first established by Wolpert [18] and was generalized by Siu [15] and Schumacher [14] to higher dimensions. A short proof can be found in [6].

To describe the curvature formulae of the Ricci and perturbed Ricci metrics, we need to introduce several operators. We first define the operator $\xi_{k}: C^{\infty}\left(X_{s}\right) \rightarrow C^{\infty}\left(X_{s}\right)$ by

$$
\xi_{k}(f)=\bar{\partial}^{*}\left(i\left(B_{k}\right) \partial f\right)=-\lambda^{-1} \partial_{z}\left(A_{k} \partial_{z} f\right)=-A_{k} K_{1} K_{0}(f)
$$

where $K_{0}, K_{1}$ are the Maass operators [18], [6].

It was proved in[6] that $\xi_{k}$ is the commutator of the Laplace operator and the Lie derivative in the direction $v_{k}$ :

$$
(\square+1) v_{k}-v_{k}(\square+1)=\square v_{k}-v_{k} \square=\xi_{k} .
$$

We also need the commutator of the operator $v_{k}$ and $\bar{v}_{l}$. In [6] we defined the operator $Q_{k \bar{l}}: C^{\infty}\left(X_{s}\right) \rightarrow C^{\infty}\left(X_{s}\right)$ by

$$
Q_{k \bar{l}}(f)=\left[\bar{v}_{l}, \xi_{k}\right](f)=\bar{P}\left(e_{k \bar{l}}\right) P(f)-2 f_{k \bar{l}} \square f+\lambda^{-1} \partial_{z} f_{k \bar{l}} \partial_{\bar{z}} f
$$

where $P: C^{\infty}\left(X_{s}\right) \rightarrow \Gamma\left(\Lambda^{1,0}\left(T^{0,1} X_{s}\right)\right)$ is the operator defined by $P(f)=$ $\partial_{z}\left(\lambda^{-1} \partial_{z} f\right)$.

The terms appeared in the curvature formulae of the Ricci and perturbed Ricci metrics are formally symmetric with respect to indices. For convenience, we recall the symmetrization operator defined in [6].

Definition 2.1. Let $U$ be any quantity which depends on indices $i, k, \alpha$, $\bar{j}, \bar{l}, \bar{\beta}$. The symmetrization operator $\sigma_{1}$ is defined by taking the summation of all orders of the triple $(i, k, \alpha)$. Similarly, $\sigma_{2}$ is the symmetrization operator of $\bar{j}$ and $\bar{\beta}$ and $\widetilde{\sigma_{1}}$ is the symmetrization operator of $\bar{j}, \bar{l}$ and $\bar{\beta}$.

Let $\widetilde{R}_{i \bar{j} k \bar{l}}$ and $P_{i \bar{j} k \bar{l}}$ be the curvature tensors of the Ricci and perturbed Ricci metrics respectively. In [6] we established the following curvature formulae of these metrics:

$$
\begin{aligned}
\widetilde{R}_{i \bar{j} k \bar{l}}= & -h^{\alpha \bar{\beta}}\left\{\sigma _ { 1 } \sigma _ { 2 } \int _ { X _ { s } } \left\{\left(T\left(\xi_{k}\left(e_{i \bar{j}}\right)\right) \bar{\xi}_{l}\left(e_{\alpha \bar{\beta}}\right)+T\left(\xi_{k}\left(e_{i \bar{j}}\right) \bar{\xi}_{\beta}\left(e_{\alpha \bar{l}}\right)\right\} d v\right\}\right.\right. \\
& -h^{\alpha \bar{\beta}}\left\{\sigma_{1} \int_{X_{s}} Q_{k \bar{l}}\left(e_{i \bar{j}}\right) e_{\alpha \bar{\beta}} d v\right\} \\
& \left.+\tau^{p \bar{q}} h^{\alpha \bar{\beta}} h^{\gamma \bar{\delta}}\left\{\sigma_{1} \int_{X_{s}} \xi_{k}\left(e_{i \bar{q}}\right) e_{\alpha \bar{\beta}} d v\right\}\left\{\widetilde{\sigma}_{1} \int_{X_{s}} \bar{\xi}_{l}\left(e_{p \bar{j}}\right) e_{\gamma \bar{\delta}}\right) d v\right\} \\
& +\tau_{p \bar{j}} h^{p \bar{q}} R_{i \bar{q} k \bar{l}}
\end{aligned}
$$


and

$$
\begin{aligned}
P_{i \bar{j} k \bar{l}}= & -h^{\alpha \bar{\beta}}\left\{\sigma_{1} \sigma_{2} \int_{X_{s}}\left\{T\left(\xi_{k}\left(e_{i \bar{j}}\right)\right) \bar{\xi}_{l}\left(e_{\alpha \bar{\beta}}\right)+T\left(\xi_{k}\left(e_{i \bar{j}}\right)\right) \bar{\xi}_{\beta}\left(e_{\alpha \bar{l}}\right)\right\} d v\right\} \\
& -h^{\alpha \bar{\beta}}\left\{\sigma_{1} \int_{X_{s}} Q_{k \bar{l}}\left(e_{i \bar{j}}\right) e_{\alpha \bar{\beta}} d v\right\} \\
& \left.+\widetilde{\tau}^{p \bar{q}} h^{\alpha \bar{\beta}} h^{\gamma \bar{\delta}}\left\{\sigma_{1} \int_{X_{s}} \xi_{k}\left(e_{i \bar{q}}\right) e_{\alpha \bar{\beta}} d v\right\}\left\{\widetilde{\sigma}_{1} \int_{X_{s}} \bar{\xi}_{l}\left(e_{p \bar{j}}\right) e_{\gamma \bar{\delta}}\right) d v\right\} \\
& +\tau_{p \bar{j}} h^{p \bar{q}} R_{i \bar{q} k \bar{l}}+C R_{i \bar{j} k \bar{l}} .
\end{aligned}
$$

It is easy to derive information of the sign of the curvature of the WeilPetersson metric from its curvature formula (2.7). However, the curvature formulae of the Ricci and perturbed Ricci metrics are too complicated to use directly. Thus we need to look at the asymptotic behavior of these metrics. We now recall geometric construction of the DM moduli space which is due to Earle-Marden and the degeneration of hyperbolic metrics. Please see [6] and [18] for details.

Let $\overline{\mathcal{M}}_{g}$ be the Deligne-Mumford compactification of $\mathcal{M}_{g}$ and let $D=$ $\overline{\mathcal{M}}_{g} \backslash \mathcal{M}_{g}$. It was shown in [2] that $D$ is a divisor with only normal crossings. A point $y \in D$ corresponds to a stable nodal surface $X_{y}$. A point $p \in X_{y}$ is a node if there is a neighborhood of $p$ which is isometric to the germ $\{(u, v) \mid u v=$ $0,|u|,|v|<1\} \subset \mathbb{C}^{2}$. Let $p_{1}, \cdots, p_{m} \in X_{y}$ be the nodes. $X_{y}$ is stable if each connected component of $X_{y} \backslash\left\{p_{1}, \cdots, p_{m}\right\}$ has negative Euler characteristic.

Fix a point $y \in D$, we assume the corresponding Riemann surface $X_{y}$ has $m$ nodes. Now for any point $s \in \mathcal{M}_{g}$ lying in a neighborhood of $y$, the corresponding Riemann surface $X_{s}$ can be decomposed into the thin part which is a disjoint union of $m$ collars and the thick part where the injectivity radius with respect to the Kähler-Einstein metric is uniformly bounded from below.

There are two kinds of local holomorphic coordinate on a collar or near a node. We first recall the rs-coordinate defined by Wolpert in [20]. In the node case, given a nodal surface $X$ with a node $p \in X$, we let $a, b$ be two punctures which are glued together to form $p$.

Definition 2.2. A local coordinate chart $(U, u)$ near $a$ is called rscoordinate if $u(a)=0$ where $u$ maps $U$ to the punctured disc $0<|u|<c$ with $c>0$, and the restriction to $U$ of the Kähler-Einstein metric on $X$ can be written as $\frac{1}{2|u|^{2}(\log |u|)^{2}}|d u|^{2}$. The rs-coordinate $(V, v)$ near $b$ is defined in a similar way. 
In the collar case, given a closed surface $X$, we assume there is a closed geodesic $\gamma \subset X$ such that its length $l=l(\gamma)<c_{*}$ where $c_{*}$ is the collar constant.

Definition 2.3. A local coordinate chart $(U, z)$ is called rs-coordinate at $\gamma$ if $\gamma \subset U$ where $z$ maps $U$ to the annulus $c^{-1}|t|^{\frac{1}{2}}<|z|<c|t|^{\frac{1}{2}}$, and the Kähler-Einstein metric on $X$ can be written as $\frac{1}{2}\left(\frac{\pi}{\log |t|} \frac{1}{|z|} \csc \frac{\pi \log |z|}{\log |t|}\right)^{2}|d z|^{2}$.

The existence of collar was due to Keen [5]. We formulate this theorem in the following:

Lemma 2.1. $\quad$ Let $X$ be a closed surface and let $\gamma$ be a closed geodesic on $X$ such that the length $l$ of $\gamma$ satisfies $l<c_{*}$. Then there is a collar $\Omega$ on $X$ with holomorphic coordinate $z$ defined on $\Omega$ such that

(1) $z$ maps $\Omega$ to the annulus $\left\{\frac{1}{c} e^{-\frac{2 \pi^{2}}{l}}<|z|<c\right\}$ for $c>0$;

(2) the Kähler-Einstein metric on $X$ restricted to $\Omega$ is given by

$$
\left(\frac{1}{2} u^{2} r^{-2} \csc ^{2} \tau\right)|d z|^{2}
$$

where $u=\frac{l}{2 \pi}, r=|z|$ and $\tau=u \log r$;

(3) the geodesic $\gamma$ is given by the equation $|z|=e^{-\frac{\pi^{2}}{l}}$;

(4) the constant $c$ has a lower bound such that the area of $\Omega$ is bounded from below by a universal constant.

We call such a collar $\Omega$ a genuine collar.

Now we describe the pinching coordinate chart of $\overline{\mathcal{M}}_{g}$ near the divisor $D$ [20]. Let $X_{0}$ be a nodal surface corresponding to a codimension $m$ boundary point and let $p_{1}, \cdots, p_{m}$ be the nodes of $X_{0}$. Then $\tilde{X}_{0}=X_{0} \backslash\left\{p_{1}, \cdots, p_{m}\right\}$ is a union of punctured Riemann surfaces. Fix rs-coordinate charts $\left(U_{i}, \eta_{i}\right)$ and $\left(V_{i}, \zeta_{i}\right)$ at $p_{i}$ for $i=1, \cdots, m$ such that all the $U_{i}$ and $V_{i}$ are mutually disjoint. Now pick an open set $U_{0} \subset \widetilde{X}_{0}$ such that the intersection of each connected component of $\widetilde{X}_{0}$ and $U_{0}$ is a nonempty relatively compact set and the intersection $U_{0} \cap\left(U_{i} \cup V_{i}\right)$ is empty for all $i$. Now pick Beltrami differentials $\nu_{m+1}, \cdots, \nu_{n}$ which are supported in $U_{0}$ and span the tangent space at $\widetilde{X}_{0}$ of the deformation space of $\widetilde{X}_{0}$. Let $\Delta_{\varepsilon}^{n-m} \subset \mathbb{C}^{n-m}$ be the polydisc of radius $\varepsilon$. For $t^{\prime \prime}=\left(t_{m+1}, \cdots, t_{n}\right) \in \Delta_{\varepsilon}^{n-m}$, let $\nu\left(t^{\prime \prime}\right)=\sum_{i=m+1}^{n} t_{i} \nu_{i}$. We assume $\left|t^{\prime \prime}\right|=\left(\sum_{i=m+1}^{n}\left|t_{i}\right|^{2}\right)^{\frac{1}{2}}$ small enough such that $\left|\nu\left(t^{\prime \prime}\right)\right|<1$. The nodal surface 
$X_{0, t^{\prime \prime}}$ is obtained by solving the Beltrami equation $\bar{\partial} w=\nu\left(t^{\prime \prime}\right) \partial w$. Since $\nu\left(t^{\prime \prime}\right)$ is supported in $U_{0},\left(U_{i}, \eta_{i}\right)$ and $\left(V_{i}, \zeta_{i}\right)$ are still holomorphic coordinates on $X_{0, t^{\prime \prime}}$. By the theory of Ahlfors and Bers [1] and Wolpert [20] we can assume that there are constants $\delta, c>0$ such that when $\left|t^{\prime \prime}\right|<\delta, \eta_{i}$ and $\zeta_{i}$ are holomorphic coordinates on $X_{0, t^{\prime \prime}}$ with $0<\left|\eta_{i}\right|<c$ and $0<\left|\zeta_{i}\right|<c$. Now we assume $t^{\prime}=\left(t_{1}, \cdots, t_{m}\right)$ has small norm. We do the plumbing construction on $X_{0, t^{\prime \prime}}$ to obtain $X_{t}=X_{t^{\prime}, t^{\prime \prime}}$. For each $i=1, \cdots, m$, we remove the discs $\{0<$ $\left.\left|\eta_{i}\right| \leq \frac{\left|t_{i}\right|}{c}\right\}$ and $\left\{0<\left|\zeta_{i}\right| \leq \frac{\left|t_{i}\right|}{c}\right\}$ from $X_{0, t^{\prime \prime}}$ and identify $\left\{\frac{\left|t_{i}\right|}{c}<\left|\eta_{i}\right|<c\right\}$ with $\left\{\frac{\left|t_{i}\right|}{c}<\left|\zeta_{i}\right|<c\right\}$ by the rule $\eta_{i} \zeta_{i}=t_{i}$. This defines the surface $X_{t}$. The tuple $t=\left(t^{\prime}, t^{\prime \prime}\right)=\left(t_{1}, \cdots, t_{m}, t_{m+1}, \cdots, t_{n}\right)$ are the local pinching coordinates for the manifold cover of $\overline{\mathcal{M}}_{g}$. We call the coordinates $\eta_{i}$ (or $\zeta_{i}$ ) the plumbing coordinates on $X_{t, s}$ and the collar $\left\{\frac{\left|t_{i}\right|}{c}<\left|\eta_{i}\right|<c\right\}$ the plumbing collar.

Remark 2.1. From the estimate of Wolpert [19], [20] on the length of short geodesic, we have $u_{i}=\frac{l_{i}}{2 \pi} \sim-\frac{\pi}{\log \left|t_{i}\right|}$.

In [6] we first proved the equivalence of canonical metrics on $\mathcal{M}_{g}$ :

Theorem 2.1. All the canonical metrics on the moduli space $\mathcal{M}_{g}$ : the Teichmüller-Kobayashi metric, the Carathéodory metric, the induced Bergman metric, the asymptotic Poincaré metric, the McMullen metric, the Ricci metric, the perturbed Ricci metric and the Kähler-Einstein metric are equivalent.

The new metrics we defined have nice curvature properties which can be used to control the Kähler-Einstein metric. In [6] and [7] we proved

Theorem 2.2. $\quad$ The Ricci and perturbed Ricci metrics are complete Kähler metrics with Poincaré growth. These metrics and the Kähler-Einstein metric have bounded geometry on the Teichmüller space $\mathcal{T}_{g}$. Furthermore, all the covariant derivatives of the curvature of the Kähler-Einstein metric are bounded. The Ricci and holomorphic sectional curvatures of the perturbed Ricci metric are bounded from above and below by negative constants.

We also derived in [6] and [7] the precise asymptotic of the Weil-Petersson, Ricci and perturbed Ricci metrics and their curvature. This is one of the key components in the proof of the goodness of these metrics. For the WeilPetersson and Ricci metrics we have

Theorem 2.3. Let $(t, s)=\left(t_{1}, \cdots, t_{m}, s_{m+1}, \cdots, s_{n}\right)$ be the pinching coordinates near a codimension $m$ boundary point in $\overline{\mathcal{M}}_{g}$. Let $h$ and $\tau$ be the Weil-Petersson and Ricci metrics respectively. Then the Weil-Petersson metric has the asymptotic: 
(1) $h^{i \bar{i}}=2 u_{i}^{-3}\left|t_{i}\right|^{2}\left(1+O\left(u_{0}\right)\right)$ and $h_{i \bar{i}}=\frac{1}{2} \frac{u_{i}^{3}}{\left|t_{i}\right|^{2}}\left(1+O\left(u_{0}\right)\right)$ for $1 \leq i \leq m$;

(2) $h^{i \bar{j}}=O\left(\left|t_{i} t_{j}\right|\right)$ and $h_{i \bar{j}}=O\left(\frac{u_{i}^{3} u_{j}^{3}}{\left|t_{i} t_{j}\right|}\right)$, if $1 \leq i, j \leq m$ and $i \neq j$;

(3) $h^{i \bar{j}}=O(1)$ and $h_{i \bar{j}}=O(1)$, if $m+1 \leq i, j \leq n$;

(4) $h^{i \bar{j}}=O\left(\left|t_{i}\right|\right)$ and $h_{i \bar{j}}=O\left(\frac{u_{i}^{3}}{\left|t_{i}\right|}\right)$ if $i \leq m<j$;

(5) $h^{i \bar{j}}=O\left(\left|t_{j}\right|\right)$ and $h_{i \bar{j}}=O\left(\frac{u_{j}^{3}}{\left|t_{j}\right|}\right)$ if $j \leq m<i$

where $u_{0}=\sum_{j=1}^{m} u_{j}+\sum_{j=m+1}^{n}\left|s_{j}\right|$. The Ricci metric has the asymptotic:

(1) $\tau_{i \bar{i}}=\frac{3}{4 \pi^{2}} \frac{u_{i}^{2}}{\left|t_{i}\right|^{2}}\left(1+O\left(u_{0}\right)\right)$ and $\tau^{i \bar{i}}=\frac{4 \pi^{2}}{3} \frac{\left|t_{i}\right|^{2}}{u_{i}^{2}}\left(1+O\left(u_{0}\right)\right)$, if $i \leq m$;

(2) $\tau_{i \bar{j}}=O\left(\frac{u_{i}^{2} u_{j}^{2}}{\left|t_{i} t_{j}\right|}\left(u_{i}+u_{j}\right)\right)$ and $\tau^{i \bar{j}}=O\left(\left|t_{i} t_{j}\right|\right)$, if $i, j \leq m$ and $i \neq j$;

(3) $\tau_{i \bar{j}}=O\left(\frac{u_{i}^{2}}{\left|t_{i}\right|}\right)$ and $\tau^{i \bar{j}}=O\left(\left|t_{i}\right|\right)$, if $i \leq m$ and $j \geq m+1$;

(4) $\tau_{i \bar{j}}=O(1)$, if $i, j \geq m+1$.

The holomorphic sectional curvature of the Ricci metric has the asymptotic:

(1) $\widetilde{R}_{i \bar{i} i \bar{i}}=-\frac{3 u_{i}^{4}}{8 \pi^{4}\left|t_{i}\right|^{4}}\left(1+O\left(u_{0}\right)\right)$ if $i \leq m$;

(2) $\widetilde{R}_{i \bar{i} i \bar{i}}=O(1)$ if $i>m$.

We also have a weak curvature estimate of the Ricci metric. Let

$$
\Lambda_{i}= \begin{cases}\frac{u_{i}}{\left|t_{i}\right|} & \text { if } i \leq m \\ 1 & \text { if } i>m .\end{cases}
$$

Then

(1) $\widetilde{R}_{i \bar{j} k \bar{l}}=O(1)$ if $i, j, k, l>m$;

(2) $\widetilde{R}_{i \bar{j} k \bar{l}}=O\left(\Lambda_{i} \Lambda_{j} \Lambda_{k} \Lambda_{l}\right) O\left(u_{0}\right)$ if at least one of these indices $i, j, k, l$ is less than or equal to $m$ and they are not all equal to each other.

The asymptotic of the perturbed Ricci metric and its curvature can be found in [6] and [7]. Also, precise estimates of the full curvature tensor of the Weil-Petersson, Ricci and perturbed Ricci metrics, which will be used in the proof of their goodness, can be found in [8] and [9]. 
Stronger estimates of the asymptotic of these metrics lead to the Mumford's goodness condition of singular Hermitian metrics on vector bundles over quasi-projective manifolds. We recall the definition and basic properties of good metrics from [11].

Let $X$ be a quasi-projective variety of $\operatorname{dim}_{\mathbb{C}} X=k$ obtained by removing a divisor $D$ of normal crossings from a closed smooth projective variety $\bar{X}$. Let $\bar{E}$ be a holomorphic vector bundle of rank $n$ over $\bar{X}$ and $E=\left.\bar{E}\right|_{X}$. Let $h$ be a Hermitian metric on $E$ which may be singular near $D$.

We cover a neighborhood of $D \subset \bar{X}$ by finitely many polydiscs

$$
\left\{U_{\alpha}=\left(\Delta^{k},\left(z_{1}, \cdots, z_{k}\right)\right)\right\}_{\alpha \in A}
$$

such that $V_{\alpha}=U_{\alpha} \backslash D=\left(\Delta^{*}\right)^{m} \times \Delta^{k-m}$. Namely, $U_{\alpha} \cap D=\left\{z_{1} \cdots z_{m}=0\right\}$. We let $U=\bigcup_{\alpha \in A} U_{\alpha}$ and $V=\bigcup_{\alpha \in A} V_{\alpha}$. On each $V_{\alpha}$ we have the local Poincaré metric

$$
\omega_{P, \alpha}=\frac{\sqrt{-1}}{2}\left(\sum_{i=1}^{m} \frac{1}{2\left|z_{i}\right|^{2}\left(\log \left|z_{i}\right|\right)^{2}} d z_{i} \wedge d \bar{z}_{i}+\sum_{i=m+1}^{k} d z_{i} \wedge d \bar{z}_{i}\right) .
$$

Definition 2.4. Let $\eta$ be a smooth local $p$-form defined on $V_{\alpha}$.

- We say $\eta$ has Poincaré growth if there is a constant $C_{\alpha}>0$ depending on $\eta$ such that

$$
\left|\eta\left(t_{1}, \cdots, t_{p}\right)\right|^{2} \leq C_{\alpha} \prod_{i=1}^{p}\left\|t_{i}\right\|_{\omega_{P, \alpha}}^{2}
$$

for any point $z \in V_{\alpha}$ and $t_{1}, \cdots, t_{p} \in T_{z} X$.

- We say $\eta$ is good if both $\eta$ and $d \eta$ have Poincaré growth.

Definition 2.5. An Hermitian metric $h$ on $E$ is good if for all $z \in V$, assuming $z \in V_{\alpha}$, and for all basis $\left(e_{1}, \cdots, e_{n}\right)$ of $\bar{E}$ over $U_{\alpha}$, if we let $h_{i \bar{j}}=$ $h\left(e_{i}, e_{j}\right)$, then

- $\left|h_{i \bar{j}}\right|,(\operatorname{det} h)^{-1} \leq C\left(\sum_{i=1}^{m} \log \left|z_{i}\right|\right)^{2 n}$ for some $C>0$ and $n \geq 1$.

- The local 1-forms $\left(\partial h \cdot h^{-1}\right)_{\alpha \gamma}$ are good on $V_{\alpha}$. Namely the local connection and curvature forms of $h$ have Poincaré growth.

It is easy to see the following basic properties of good metrics:

- The definition of Poincaré growth is independent of the choice of $U_{\alpha}$ or local coordinates on it. 
- A form $\eta \in A^{p}(X)$ with Poincaré growth defines a $p$-current $[\eta]$ on $\bar{X}$. In fact we have

$$
\int_{X}|\eta \wedge \xi|<\infty
$$

for any $\xi \in A^{k-p}(\bar{X})$.

- If both $\eta \in A^{p}(X)$ and $\xi \in A^{q}(X)$ have Poincaré growth, then $\eta \wedge \xi$ has Poincaré growth.

- For a good form $\eta \in A^{p}(X)$, we have $d[\eta]=[d \eta]$.

The importance of a good metric on $E$ is that we can compute the Chern classes of $\bar{E}$ via the Chern forms of $h$ as currents. Namely, with the growth assumptions on the metric and its derivatives, we can integrate by part, so Chern-Weil theory still holds. In [11] Mumford has proved:

Theorem 2.4. Given an Hermitian metric $h$ on $E$, there is at most one extension $\bar{E}$ of $E$ to $\bar{X}$ such that $h$ is good.

Theorem 2.5. If $h$ is a good metric on $E$, the Chern forms $c_{i}(E, h)$ are good forms. Furthermore, as currents, they represent the corresponding Chern classes $c_{i}(\bar{E}) \in H^{2 i}(\bar{X}, \mathbb{C})$.

In the following sections, we will discuss the goodness of the above metrics and their applications.

\section{$\S 3 . \quad$ Goodness of the Weil-Petersson Metric}

From Theorem 2.3, it is very natural to consider the metrics induced by the Weil-Petersson, Ricci, perturbed Ricci and Kähler-Einstein metrics on the logarithmic extension $\bar{E}=T_{\mathcal{M}_{g}}^{*}(\log D)$ of the cotangent bundle $T_{\mathcal{M}_{g}}^{*}$ to the DM moduli space $\overline{\mathcal{M}}_{g}$.

We first give a general discussion of the goodness condition of the metric on $\bar{E}$ induced by a Kähler metric $g$ on $\mathcal{M}_{g}$. Let $D=\overline{\mathcal{M}}_{g} \backslash \mathcal{M}_{g}$ be the compactification divisor and let $p \in D$ be a codimension $m$ boundary point in $\overline{\mathcal{M}}_{g}$ with the corresponding stable nodal surface $X_{0,0}$. Let $n=3 g-3$ be the dimension of $\mathcal{M}_{g}$. Let $\left(t_{1}, \cdots, t_{n}\right)$ be the pinching coordinates near $p$ where $\left(t_{1}, \cdots, t_{m}\right)$ corresponding to the degeneration directions.

For any Kähler metric $g$ on $\mathcal{M}_{g}$, let $g^{*}$ be the induced metric on $\bar{E}$. We know that

$$
\left(\frac{d t_{1}}{t_{1}}, \cdots, \frac{d t_{m}}{t_{m}}, d t_{m+1}, \cdots, d t_{n}\right)
$$


is a local holomorphic frame of $\bar{E}$. Under this frame, the metric $g^{*}$ and its inverse are given by

$$
g_{i \bar{j}}^{*}= \begin{cases}\frac{1}{t_{i} \bar{t}_{j}} g^{i \bar{j}} & i, j \leq m \\ \frac{1}{t_{i}} g^{i \bar{j}} & i \leq m<j \\ \frac{1}{\bar{t}_{j}} g^{i \bar{j}} & j \leq m<i \\ g^{i \bar{j}} & i, j>m\end{cases}
$$

and

$$
\left(g^{*}\right)^{i \bar{j}}= \begin{cases}t_{i} \bar{t}_{j} g_{i \bar{j}} & i, j \leq m \\ t_{i} g_{i \bar{j}} & i \leq m<j \\ \bar{t}_{j} g_{i \bar{j}} & j \leq m<i \\ g_{i \bar{j}} & i, j>m .\end{cases}
$$

Now we define two quantities. Let

$$
D_{i}^{k}= \begin{cases}\frac{t_{k}}{t_{i}} & i, k \leq m \\ t_{k} & k \leq m<i \\ \frac{1}{t_{i}} & i \leq m<k \\ 1 & i, k>m .\end{cases}
$$

Let $v_{i}=-\frac{\pi}{\log \left|t_{i}\right|}$ for $i \leq m$ and let $u_{i}=\frac{l_{i}}{2 \pi}$ where $l_{i}$ is the length of the geodesic loop on the $i$-th collar of $X=X_{t}$. We have that

$$
u_{i}=v_{i}\left(1+O\left(v_{i}\right)\right) \text {. }
$$

Now we let

$$
\Lambda_{i}= \begin{cases}\frac{u_{i}}{\left|t_{i}\right|} & i \leq m \\ 1 & i>m\end{cases}
$$

Let $A=\left(A_{i}^{k}\right)$ be the connection form of $g^{*}$ where $i$ is the row index and $k$ is the column index. We have

$$
A_{i}^{k}=\sum_{p}\left(\sum_{j}\left(\partial_{p} g_{i \bar{j}}^{*}\right)\left(g^{*}\right)^{k \bar{j}}\right) d t_{p} .
$$

By (3.2), (3.3) and (3.4), we have

$$
A_{i}^{k}=-\sum_{p}\left(D_{i}^{k} \sum_{j}\left(\partial_{p} g_{k \bar{j}}\right) g^{i \bar{j}}\right) d t_{p}
$$


if $i \neq k$ or $i=k>m$. We also have

$$
A_{i}^{i}=-\sum_{p \neq i}\left(\sum_{j}\left(\partial_{p} g_{i \bar{j}}\right) g^{i \bar{j}}\right) d t_{p}-\left(\frac{1}{t_{i}}+\sum_{j}\left(\partial_{i} g_{i \bar{j}}\right) g^{i \bar{j}}\right) d t_{i}
$$

if $i=k \leq m$.

To prove the goodness of $g^{*}$, the first order estimates are reduced to

$$
\left|D_{i}^{k} \sum_{j}\left(\partial_{p} g_{k \bar{j}}\right) g^{i \bar{j}}\right|=O\left(\Lambda_{p}\right)
$$

if $i \neq k$ or $i=k>m$ or $p \neq i=k \leq m$ and

$$
\left|\frac{1}{t_{i}}+\sum_{j}\left(\partial_{i} g_{i \bar{j}}\right) g^{i \bar{j}}\right|=O\left(\Lambda_{i}\right)
$$

if $i=k=p \leq m$.

For the estimates on the $g^{*}$ itself, we need to show that

$$
\left|g_{i \bar{j}}^{*}\right|,\left(\operatorname{det} g^{*}\right)^{-1} \leq C\left(\sum_{i=1}^{m} \log \left|t_{i}\right|\right)^{2 n} .
$$

By (3.2) we have $\left(\operatorname{det} g^{*}\right)^{-1}=\left|t_{1} \cdots t_{m}\right|^{2}(\operatorname{det} g)$, inequality (3.11) is equivalent to

$$
\left|g_{i \bar{j}}^{*}\right|,\left|t_{1} \cdots t_{m}\right|^{2}(\operatorname{det} g) \leq C\left(\sum_{i=1}^{m} \log \left|t_{i}\right|\right)^{2 n} .
$$

The second order estimates are reduced to show that $d A_{i}^{k}$ has Poincaré growth for any choice of $i, k$. Since

$$
d A=\partial A+\bar{\partial} A=\bar{\partial} A-A \wedge A,
$$

if each entry of $A$ has Poincaré growth, then each entry of $A \wedge A$ has Poincaré growth. Thus we need to show that each entry of $\bar{\partial} A$ has Poincaré growth.

By (3.7) and (3.8), since $D_{i}^{i}=1$, we have

$$
\bar{\partial} A_{i}^{k}=D_{i}^{k} \partial_{\bar{q}}\left(\sum_{j}\left(\partial_{p} g_{k \bar{j}}\right) g^{i \bar{j}}\right) d t_{p} \wedge d \bar{t}_{q}=-D_{i}^{k} g^{i \bar{j}} R_{k \bar{j} p \bar{q}} d t_{p} \wedge d \bar{t}_{q}
$$

where $R_{k \bar{j} p \bar{q}}$ is the curvature of $g$. Thus we need to show that

$$
\left|D_{i}^{k} g^{i \bar{j}} R_{k \bar{j} p \bar{q}}\right|=O\left(\Lambda_{p} \Lambda_{q}\right) .
$$

By collecting the above argument, we have 
Lemma 3.1. The metric $g^{*}$ on $T_{\mathcal{M}_{g}}^{*}(\log D)$ induced by a Kähler metric $g$ on $\mathcal{M}_{g}$ is good if and only if the estimates (3.9), (3.10), (3.12) and (3.14) hold.

In this section, we will focus on the goodness of the metric induced by the Weil-Petersson metric $h$. The main theorem is

Theorem 3.1. The metric $h^{*}$ on the logarithm cotangent bundle $\bar{E}$ over the DM moduli space induced by the WP metric is good in the sense of Mumford. Thus the Chern forms of $h^{*}$, as currents, are equal to the Chern classes of $\bar{E}$.

We now sketch the proof of this theorem in three steps: the zero-th order, first order and second order estimates. The details are in [8]. In the following, we take the metric $g_{i \bar{j}}$ to be the Weil-Petersson metric $h_{i \bar{j}}$. We use the same notation as in our paper [6].

We first consider the zero-th order estimate. This follows directly from Theorem 2.3.

Lemma 3.2. The inequality (3.12) hold for the Weil-Petersson metric $h$.

Proof. By Theorem 2.3 and (3.2), (3.3), we have

$$
h_{i \bar{j}}^{*}= \begin{cases}2 u_{i}^{-3}\left(1+O\left(u_{0}\right)\right) & i=j \leq m \\ O(1) & \text { otherwise }\end{cases}
$$

and

$$
\left|t_{1} \cdots t_{m}\right|^{2}(\operatorname{det} h) \leq C\left(\prod_{i=1}^{m} u_{i}\right)^{3} .
$$

It is easy to see that (3.12) hold.

Now we prove the first order estimates. In order to compute the connection of the induced metric $h^{*}$, it is easier to prove the formula (3.6) directly with the metric $h$. We use the estimate of Masur [10] and refine the estimates of Schumacher [13] and Trapani [16].

By the work of [6] we know that

$$
h^{i \bar{j}}=\int_{X} \frac{\varphi_{i} \bar{\varphi}_{j}}{\lambda^{2}} d v
$$

where $\varphi_{i}$ is the holomorphic quadratic differential corresponding to $d t_{i}$ and $\lambda$ is the KE metric on $X$. In order to compute the connection forms of the WP metric, we need to estimate the derivatives of each $\varphi_{i}$ and $\lambda$. 
Unlike the approach in [6], here we take plumbing coordinate and plumbing collar rather than rs-coordinate and genuine collar because we need the trivialization of the collars. It is easier to compute the derivative of the hyperbolic metric by using the plumbing coordinate on the degeneration collars.

We first change coordinate on the collars. Let $\left(t_{1}, \cdots, t_{n}\right)$ be the pinching coordinates near a codimension $m$ boundary point $p$ in the DM moduli. Let $z_{i}$ and $w_{i}$ be the plumbing coordinates on the $i$-th collar of $X_{t}$ with $i \leq m$. Let $r_{i}=\left|z_{i}\right|, \theta_{i}=\arg z_{i}, \widetilde{r}_{i}=\left|w_{i}\right|$ and $\widetilde{\theta}_{i}=\arg w_{i}$. We know that $z_{i} w_{i}=t_{i}$. Let $\Omega_{c}^{i}$ be the $i$-th plumbing collar of size $c$ with a fixed $0<c<1$. Namely,

$$
\Omega_{c}^{i}=\left\{z_{i}\left|c^{-1}\right| t_{i} \mid \leq r_{i} \leq c\right\}=\left\{w_{i}\left|c^{-1}\right| t_{i} \mid \leq \widetilde{r}_{i} \leq c\right\}
$$

We denote by $\Omega_{c}$ the union of all collars: $\Omega_{c}=\bigcup_{i=1}^{m} \Omega_{c}^{i}$. We also define the half collars $\Omega_{c}^{i+}$ and $\Omega_{c}^{i-}$ by

$$
\Omega_{c}^{i+}=\left\{\left.z_{i}|| t_{i}\right|^{\frac{1}{2}} \leq r_{i} \leq c\right\}
$$

and

$$
\Omega_{c}^{i-}=\left\{\left.z_{i}\left|c^{-1}\right| t_{i}\left|\leq r_{i} \leq\right| t_{i}\right|^{\frac{1}{2}}\right\}=\left\{\left.w_{i}|| t_{i}\right|^{\frac{1}{2}} \leq \widetilde{r}_{i} \leq c\right\} .
$$

To compute the derivative of $\varphi$, by our works in [6] and the work of Masur [10], we have the expansion of $\varphi_{i}$ on the plumbing collars. Let $\Delta_{\delta}^{n}$ be the closed polydisc in $\mathbb{C}^{n}$ such that the radius of each disk is $\delta>0$. We assume the pinching coordinates $t=\left(t_{1}, \cdots, t_{n}\right)$ is defined for $t \in \Delta_{\delta}^{n}$. By shrinking $\delta$ we have

Lemma 3.3. Let $k \leq m$ and let $z_{k}$ and $w_{k}$ be the plumbing coordinates on the $k$-th collar $\Omega_{c_{0}}^{k}$ with $c<c_{0}<1$ fixed. Then on $\Omega_{c_{0}}^{k}$ we have

(1) $\varphi_{i}=-\frac{t_{i}}{\pi} \frac{1}{z_{k}^{2}}\left(p_{i}^{k}\left(z_{k}\right)+q_{i}^{k}\left(z_{k}\right)\right)$ if $i \leq m$ and $i \neq k$;

(2) $\varphi_{i}=\frac{1}{z_{k}^{2}}\left(p_{i}^{k}\left(z_{k}\right)+q_{i}^{k}\left(z_{k}\right)\right)$ if $i>m$

(3) $\varphi_{k}=-\frac{t_{k}}{\pi} \frac{1}{z_{k}^{2}}\left(1+p_{k}^{k}\left(z_{k}\right)+q_{k}^{k}\left(z_{k}\right)\right)$.

There is a constant $M>0$ such that in the above formulae, the functions $p_{i}^{k}, q_{i}^{k}$ satisfy

(1) $p_{i}^{k}=\sum_{s=1}^{\infty} a_{i s}^{k}(t) z_{k}^{s}$ such that each $a_{i s}^{k}(t)$ is a holomorphic function of the multi-variable $t$ and $\sum_{s=1}^{\infty}\left|a_{i s}^{k}(t)\right| c_{0}^{s} \leq M$ for $t \in \Delta_{\delta}^{n}$;

(2) $q_{i}^{k}=\sum_{s<-1} a_{i s}^{k}(t) t_{k}^{-s} z_{k}^{s}$ such that $a_{i s}^{k}(t)$ is holomorphic in $t$ and $\sum_{s \leq-1}\left|a_{i s}^{k}(\bar{t})\right| c_{0}^{-s} \leq M$ for $t \in \Delta_{\delta}^{n}$. 
There are similar expansions by using the $w_{k}$ coordinates. Furthermore, on $X \backslash \Omega_{c}$ we have

$$
\left\|\varphi_{i}\right\|= \begin{cases}O\left(\left|t_{i}\right|\right) & i \leq m \\ O(1) & i>m\end{cases}
$$

For the proof of this lemma, please see [10]. We also have the estimates of the derivatives of $p_{i}^{k}$ and $q_{i}^{k}$ :

Lemma 3.4. Let $0<c<c_{0}$ be a fixed constant. On the collar $\Omega_{c}^{k}$ we have

(1) $\frac{\partial p_{i}^{k}}{\partial t_{j}}=\sum_{s=1}^{\infty} \frac{\partial a_{i s}^{k}(t)}{\partial t_{j}} z_{k}^{s}$ such that $\sum_{s=1}^{\infty}\left|\frac{\partial a_{i s}^{k}(t)}{\partial t_{j}}\right| c^{s} \leq M_{1}$ for $t \in \Delta_{\frac{\delta}{2}}^{n}$;

(2) $\frac{\partial q_{i}^{k}}{\partial t_{j}}=\sum_{s \leq-1} \frac{\partial a_{i s}^{k}(t)}{\partial t_{j}} t_{k}^{-s} z_{k}^{s}$ such that $\sum_{s \leq-1}\left|\frac{\partial a_{i s}^{k}(t)}{\partial t_{j}}\right| c^{-s} \leq M_{1}$ for $t \in \Delta_{\frac{\delta}{2}}^{n}$ and $j \neq k$;

(3) $\frac{\partial q_{i}^{k}}{\partial t_{k}}=\frac{1}{t_{k}} \sum_{s \leq-1} b_{i s}^{k}(t) t_{k}^{-s} z_{k}^{s}$ where $b_{i s}^{k}(t)=t_{k} \frac{\partial a_{i s}^{k}(t)}{\partial t_{k}}-s a_{i s}^{k}(t)$ and $\sum_{s \leq-1}\left|b_{i s}^{k}(t)\right| c^{-s} \leq M_{1}$.

Here $M_{1}$ is a constant depending on $M, c, c_{0}, \delta, n$.

By combining the above two lemmas, we can get desired estimates of the derivatives of each quadratic differential $\varphi_{i}$.

We then estimate the KE metric $\lambda$ and its derivatives on each Riemann surface. The following estimate of $\lambda$ is due to Masur [10]. The following lemma, although is not sharp, will be enough for our purpose.

Lemma 3.5. For each $1 \leq i \leq m$, there is a constant $\alpha>0$ such that, on $\Omega_{c}^{i+}$, we have

$$
\frac{1}{\alpha} \frac{1}{r_{i}^{2}\left(\log r_{i}\right)^{2}} \leq \lambda \leq \alpha \frac{1}{r_{i}^{2}\left(\log r_{i}\right)^{2}}
$$

and on $\Omega_{c}^{i-}$, we have

$$
\frac{1}{\alpha} \frac{1}{\widetilde{r}_{i}^{2}\left(\log \widetilde{r}_{i}\right)^{2}} \leq \lambda \leq \alpha \frac{1}{\widetilde{r}_{i}^{2}\left(\log \widetilde{r}_{i}\right)^{2}} .
$$

The estimate the derivative of $\lambda$ is more subtle. We have

Lemma 3.6. Let $\lambda$ be the KE metric on the Riemann surface $X=X_{t}$. On each collar $\Omega_{c}^{k}, \lambda$ has a unique representation in term of the plumbing coordinate $z_{k}$. Then

$$
\left|\frac{\partial}{\partial t_{i}}\left(\left.\log \lambda\right|_{\Omega_{c}}\right)\right|=O\left(\Lambda_{i}\right)
$$


Some ideas of the proof of this lemma was drawn from the work of Schumacher in [13]. We briefly describe the proof here by using the compound graft metric constructed by Wolpert in [20].

Let $\mathfrak{X}$ be the total space and let $\pi: \mathfrak{X} \rightarrow \mathcal{M}_{g}$ be the projection. In [6], we established the curvature formulae of the WP metric and the Ricci metric by using the harmonic lift which directly gives the harmonic representatives of the Kodaira-Spencer classes. In this case, we need to use a different lift. For each $i$, let $v_{i}$ be the harmonic lift of $\frac{\partial}{\partial t_{i}}$. Let $\widetilde{v}_{i}$ be a lift of $\frac{\partial}{\partial t_{i}}$ such that $\left.\widetilde{v}_{i}\right|_{\pi^{-1}\left(\Omega_{c}\right)}=\frac{\partial}{\partial t_{i}}$. This can be done since we have a trivialization of $\pi^{-1}\left(\Omega_{c}\right)$ by using the plumbing coordinates on the collars. $\widetilde{v}_{i}$ can be obtained by gluing fiberwisely an appropriately chosen lift of $\frac{\partial}{\partial t_{i}}$ on $X \backslash \Omega_{c}$ and the vector field $\frac{\partial}{\partial t_{i}}$ on $\Omega_{c}$ using a graft function.

Now let $\tilde{\lambda}$ be the compound graft metric on $X$. A direct computation shows that

$$
\left|\frac{L_{\widetilde{v}_{i}} \tilde{\lambda}}{\widetilde{\lambda}}-\frac{L_{\widetilde{v}_{i}} \lambda}{\lambda}\right|=O\left(\Lambda_{i}\right)
$$

and

$$
\left|\frac{L_{\widetilde{v}_{i}} \widetilde{\lambda}}{\widetilde{\lambda}}\right|=O\left(\Lambda_{i}\right) .
$$

The above two formulae imply

$$
\left|\frac{L_{\widetilde{v}_{i}} \lambda}{\lambda}\right|=O\left(\Lambda_{i}\right)
$$

which is the conclusion since $\left.\widetilde{v}_{i}\right|_{\pi^{-1}\left(\Omega_{c}\right)}=\frac{\partial}{\partial t_{i}}$.

The estimates on $\varphi_{i}, \lambda$ and their derivatives give the estimates on the derivatives of the dual metric of the WP metric:

Lemma 3.7. Let $t=\left(t_{1}, \cdots, t_{n}\right)$ be the local pinching coordinates with $t \in \Delta_{\delta}^{n}$. Assume $\delta$ is small enough. If $p>m$, then

$$
\left|\partial_{p} h^{i \bar{j}}\right|= \begin{cases}O\left(\left|t_{i}\right|^{2} u_{i}^{-3}\right) & i=j \leq m \\ O\left(\left|t_{i} t_{j}\right|\right) & i, j \leq m, i \neq j \\ O\left(\left|t_{i}\right|\right) & i \leq m<j \\ O\left(\left|t_{j}\right|\right) & j \leq m<i \\ O(1) & i, j>m .\end{cases}
$$


If $p \leq m$ and $p \neq i$, then

$$
\left|\partial_{p} h^{i \bar{j}}\right|= \begin{cases}O\left(\left|t_{i}\right|^{2} u_{i}^{-3} \Lambda_{p}\right) & i=j \leq m \\ O\left(\left|t_{i} t_{j}\right| \Lambda_{p}\right) & i, j \leq m, i \neq j \\ O\left(\left|t_{i}\right| \Lambda_{p}\right) & i \leq m<j \\ O\left(\left|t_{j}\right| \Lambda_{p}\right) & j \leq m<i \\ O\left(\Lambda_{p}\right) & i, j>m .\end{cases}
$$

If $p=i \leq m$ then

$$
\left|\partial_{i}\left(\frac{1}{t_{i}} h^{i \bar{j}}\right)\right|= \begin{cases}O\left(u_{i}^{-2}\right) & i=j \leq m \\ O\left(\left|\frac{t_{j}}{t_{i}}\right| u_{i}^{-2}\right) & j \leq m, i \neq j \\ O\left(\left|t_{i}\right|^{-1} u_{i}^{-2}\right) & j>m .\end{cases}
$$

We omit the proof of this lemma since it consists of very technique estimates. Please see [8] for details. Now the first order estimate follows from the above lemma, Theorem 2.3 and direct computations:

Lemma 3.8. Let $h^{*}$ be the metric on $\bar{E}$ induced by the WP metric. Then

$$
\left|\sum_{j} \partial_{p}\left(h_{i \bar{j}}^{*}\right)\left(h^{*}\right)^{k \bar{j}}\right|=O\left(\Lambda_{p}\right)
$$

for any $i$ and $k$.

Remark 3.1. Let $\Gamma_{i j}^{k}$ be the Christoffell symbol of the WP metric under the pinching coordinates. By (3.9) and (3.10), Lemma 3.8 is equivalent to

(1) $\left|\Gamma_{i i}^{i}+\frac{1}{t_{i}}\right|=O\left(\Lambda_{i}\right)$ if $p=i=k \leq m$;

(2) $\left|D_{i}^{k} \Gamma_{k p}^{i}\right|=O\left(\Lambda_{p}\right)$ otherwise.

Finally we briefly discuss the second order estimate. We only give the main steps and omit the details. On one hand, since we need to estimate the curvature tensor of the Weil-Petersson metric, we can use the techniques in [6]. On the other hand, we need precise estimates which require us to repeat the work in [6] in an optimal way.

First of all, by Theorem 2.3, formula (3.14) is equivalent to the following two formulae:

$$
\left|R_{k \bar{j} p \bar{q}}\right|= \begin{cases}O\left(\Lambda_{p} \Lambda_{q} \frac{u_{j}^{3}}{\left|t_{j}\right|}\right) & j \leq m \\ O\left(\Lambda_{p} \Lambda_{q}\right) & j>m\end{cases}
$$


if $k>m$ and

$$
\left|R_{k \bar{j} p \bar{q}}\right|= \begin{cases}O\left(\Lambda_{p} \Lambda_{q} \frac{u_{j}^{3}}{\left|t_{j} t_{k}\right|}\right) & j \leq m \\ O\left(\Lambda_{p} \Lambda_{q} \frac{1}{\left|t_{k}\right|}\right) & j>m\end{cases}
$$

if $k \leq m$.

To check these two formulae, we use the curvature formula of the WeilPetersson metric:

$$
R_{k \bar{j} p \bar{q}}=-\int_{X}\left(e_{k \bar{j}} f_{p \bar{q}}+e_{k \bar{q}} f_{p \bar{j}}\right) d v .
$$

In this case, we will use rs-coordinates on the genuine collars. We let $X=X_{t}$ and let $\Omega_{c}^{i}$ be the $i$-th genuine collar in $X$ of size $c$. Let $z_{i}$ be a rs-coordinate on $\Omega_{c}^{i}$ with $r_{i}=\left|z_{i}\right|$ and let $\rho_{i}=e^{-\frac{2 \pi^{2}}{l_{i}}}$. By [6] we know that

$$
\Omega_{c}^{i}=\left\{z_{i} \mid c^{-1} \rho_{i} \leq r_{i} \leq c\right\}
$$

We define the half collars

$$
\Omega_{c}^{i+}=\left\{z_{i} \mid \rho_{i}^{\frac{1}{2}} \leq r_{i} \leq c\right\}
$$

and

$$
\Omega_{c}^{i-}=\left\{z_{i} \mid c^{-1} \rho_{i} \leq r_{i} \leq \rho_{i}^{\frac{1}{2}}\right\} .
$$

For $0<c_{1}<c$, we define $\Omega_{c}^{j} \backslash \Omega_{c_{1}}^{j}=\Gamma_{1}^{j} \cup \Gamma_{2}^{j}$ where

$$
\Gamma_{1}^{j}=\left\{c_{1}<r_{j} \leq c\right\}
$$

and

$$
\Gamma_{2}^{j}=\left\{c^{-1} \rho_{j} \leq r_{j}<c_{1}^{-1} \rho_{j}\right\}
$$

Let the functions $\widetilde{e}_{i \bar{j}}$ be defined as in [9]. The following technical lemma, as an optimal version of the proof of Corollary 4.2 of [6], is one of the key ingredient of the second order estimate.

Lemma 3.9. Let $j \leq m$. Then $\int_{\Omega_{c_{1}}^{j}} \widetilde{e}_{j \bar{j}} f_{k \bar{k}} d v \geq 0$ and

$$
\int_{\Omega_{c_{1}}^{j}} \widetilde{e}_{j \bar{j}} f_{k \bar{k}} d v= \begin{cases}O\left(\frac{u_{j}^{4}}{\left|t_{j}\right|^{2}}\right) & k>m \\ O\left(\frac{u_{j}^{4}}{\left|t_{j}\right|^{2}} \frac{u_{k}^{6}}{\left|t_{k}\right|^{2}}\right) & k \leq m, k \neq j \\ O\left(\frac{u_{j}^{5}}{\left|t_{j}\right|^{4}}\right) & k=m .\end{cases}
$$

By using this lemma, we have the second order estimate: 
Lemma 3.10. The formulae (3.19) and (3.20) hold.

The proof of this part follows from Lemma 3.9 and a detailed case by case check. See [8]. Now we are ready to prove the main theorem 3.1.

Proof. By Lemmas 3.2, 3.8 and 3.10 we already checked the goodness condition of the metric $h^{*}$ by using the frame (3.1). If we choose another local holomorphic frame of $\bar{E}$, it is clear that the change matrix is holomorphic and its determinant is non-zero and bounded. Thus the zero order, first order and second order estimates of using the new frame differ from the estimates of using the frame (3.1) by bounded terms. We finish the proof.

\section{§4. Dual Nakano Negativity of the Weil-Petersson Metric}

The Weil-Petersson metric has many negative curvature properties. Ahlfors showed that its Riemannian sectional curvature is negative. Later, Schumacher showed in [13] that the curvature of the WP metric is strongly negative in the sense of Siu. In this section, we prove that WP metric is dual Nakano negative from which we will derive Nakano-type vanishing theorems in next section.

We first recall the concept of dual Nakano negativity. Let $\left(E^{m}, h\right)$ be a holomorphic vector bundle with a Hermitian metric over a complex manifold $M^{n}$. The curvature of $E$ is given by

$$
P_{i \bar{j} \alpha \bar{\beta}}=-\partial_{\alpha} \partial_{\bar{\beta}} h_{i \bar{j}}+h^{p \bar{q}} \partial_{\alpha} h_{i \bar{q}} \partial_{\bar{\beta}} h_{p \bar{j}} .
$$

$(E, h)$ is Nakano semi-positive if the curvature $P$ defines a semi-positive form on the bundle $E \otimes T_{M}$. Namely,

$$
P_{i \bar{j} \alpha \bar{\beta}} C^{i \alpha} \overline{C^{j \beta}} \geq 0
$$

for all $m \times n$ complex matrix $C$. The metric $h$ is Nakano positive if (4.1) is a strict inequality whenever $C \neq 0 . E$ is dual Nakano (semi) negative if the dual bundle with the induced metric $\left(E^{*}, h^{*}\right)$ is Nakano (semi) positive.

Our main result in this section is

Theorem 4.1. Let $\mathcal{M}_{g}$ be the moduli space of Riemann surfaces of genus $g \geq 2$. Then $\left(T_{\mathcal{M}_{g}}, \omega_{W P}\right)$ is dual Nakano negative.

To prove that the WP metric $h$ on the tangent bundle of $\mathcal{M}_{g}$ is Nakano negative, we only need to show that $\left(T^{*} \mathcal{M}_{g}, h^{*}\right)$ is Nakano positive. Let $R_{i \bar{j} k \bar{l}}$ 
be the curvature of $\mathcal{M}_{g}$ and $P_{i \bar{j} k \bar{l}}$ be the curvature of the cotangent bundle. We first have

$$
P_{m \bar{n} k \bar{l}}=-h^{i \bar{n}} h^{m \bar{j}} R_{i \bar{j} k \bar{l}} .
$$

Thus if we let $a_{k j}=\sum_{m} h^{m \bar{j}} C^{m k}$, we have

$$
P_{m \bar{n} k \bar{l}} C^{m k} \overline{C^{n l}}=-\sum_{i, j, k, l} R_{i \bar{j} k \bar{l}} a_{k j} \overline{a_{l i}}=-\sum_{i, j, k, l} R_{k \bar{j} i \bar{l}} a_{k j} \overline{a_{l i}}=-\sum_{i, j, k, l} R_{i \bar{j} k \bar{l}} a_{i j} \overline{a_{l k}} .
$$

Recall that at $X \in \mathcal{M}_{g}$ we have

$$
R_{i \bar{j} k \bar{l}}=-\int_{X}\left(e_{i \bar{j}} f_{k \bar{l}}+e_{i \bar{l}} f_{k \bar{j}}\right) d v .
$$

By combining the above two formulae, to prove that the WP metric is Nakano negative is equivalent to show that

$$
\int_{X}\left(e_{i \bar{j}} f_{k \bar{l}}+e_{i \bar{l}} f_{k \bar{j}}\right) a_{i j} \overline{a_{l k}} d v \geq 0
$$

and the left hand side of the above formula is strictly positive if $A=\left[a_{i j}\right] \neq 0$.

We now describe the proof with the assumption that the matrix $\left[a_{i j}\right]$ is invertible. The general case can be found in [8] which follows from the same idea.

Recall that if we let $\square=-\lambda^{-1} \partial_{z} \partial_{\bar{z}}$ be the Laplace operator with respect to the KE metric $\lambda$ on $X$ and let $T=(\square+1)^{-1}$, then $e_{i \bar{j}}=T\left(f_{i \bar{j}}\right)$ where $f_{i \bar{j}}=A_{i} \overline{A_{j}}$ and $A_{i}$ is the harmonic representative of the Kodaira-Spencer class of $\frac{\partial}{\partial t_{i}}$ where $\left(t_{1}, \cdots, t_{n}\right)$ are local coordinates on $\mathcal{M}_{g}$ and $z$ is the local coordinate on $X_{t}$.

Let $B_{j}=\sum_{i=1}^{n} a_{i j} A_{i}$. Then the inequality (4.2) is equivalent to

$$
\begin{aligned}
& -\sum_{j, k} R\left(B_{j}, \overline{B_{k}}, A_{k}, \overline{A_{j}}\right) \\
= & \sum_{j, k} \int_{X}\left(T\left(B_{j} \overline{A_{j}}\right) A_{k} \overline{B_{k}}+T\left(B_{j} \overline{B_{k}}\right) A_{k} \overline{A_{j}}\right) d v \geq 0 .
\end{aligned}
$$

Since $\left\{A_{k}\right\}$ is a basis of the space $H^{0,1}\left(X, T_{X}\right)$ and the matrix $\left\{a_{i j}\right\}$ is an arbitrary invertible matrix, we need to show that the inequality (4.3) holds for any two bases $\left\{A_{i}\right\}$ and $\left\{B_{i}\right\}$. Of course we can choose one basis, say $\left\{A_{i}\right\}$, and let the other basis vary freely.

Now we prove the inequality (4.3). Let $\mu=\sum_{j} B_{j} \overline{A_{j}}$. Then the first term in $(4.3)$ is

$$
\sum_{j, k} \int_{X} T\left(B_{j} \overline{A_{j}}\right) A_{k} \overline{B_{k}} d v=\int_{X} T(\mu) \bar{\mu} d v \geq 0 .
$$


To check the second term, we let $G(z, w)$ be the Green's function of the operator $T$. Namely, for any function $f \in C^{\infty}(X)$, we have $T(f)=$ $\int_{X} G(z, w) f(w) d v(w)$. Now we let

$$
H(z, w)=\sum_{j} \overline{A_{j}}(z) B_{j}(w)
$$

We know the second term of (4.3) is

$$
\begin{aligned}
\sum_{j, k} \int_{X} T\left(B_{j} \overline{B_{k}}\right) A_{k} \overline{A_{j}} d v & =\sum_{j, k} \int_{X} \int_{X} G(z, w) B_{j}(w) \overline{B_{k}}(w) A_{k}(z) \overline{A_{j}}(z) d v(w) d v(z) \\
& =\int_{X} \int_{X} G(z, w) H(z, w) \bar{H}(z, w) d v(w) d v(z) \geq 0
\end{aligned}
$$

where the last inequality follows from the fact that the Green's function $G$ is non-negative which is proved by Wolpert in [18, page 136].

Here we note that $H(z, w)$ is not a global function. However, we can overcome this by using a simple partition of unity argument. See [8] for details.

Remark 4.1. It is possible to show that the singular metric on $T_{\overline{\mathcal{M}}_{g}}(-\log D)$ induced by the Weil-Petersson metric is also dual Nakano negative. This follows from the dual Nakano negativity of the WP metric and simple linear algebra.

\section{$\S 5 . \quad L^{2}$-Cohomology and Rigidity}

The dual Nakano negativity of a Hermitian metric on a bundle over a compact manifold gives strong vanishing theorems by using Bochner techniques. However, in our case the base variety $\mathcal{M}_{g}$ is only quasi-projective. Thus we can only describe vanishing theorems of the $L^{2}$ cohomology.

In [12], Saper showed that the $L^{2}$ cohomology of the moduli space equipped with the Weil-Petersson metric can be identified with the ordinary cohomology of the DM moduli space. Our situation is more subtle since the natural object to be considered in our case is the tangent bundle valued $L^{2}$ cohomology. Parallel to Saper's work, we proved in [8]

Theorem 5.1. We have the following natural isomorphism:

$$
H_{(2)}^{*}\left(\left(\mathcal{M}_{g}, \omega_{\tau}\right),\left(T_{\mathcal{M}_{g}}, \omega_{W P}\right)\right) \cong H^{*}\left(\overline{\mathcal{M}}_{g}, T_{\overline{\mathcal{M}}_{g}}(-\log D)\right)
$$

where $\omega_{\tau}$ is the Ricci metric on $\mathcal{M}_{g}$. 
We first explain this theorem. To define the $L^{2}$ cohomology of a vector bundle over a manifold, we need metrics on both the bundle and the manifold. Here we view $T_{\mathcal{M}_{g}}$ purely as a bundle over the moduli space $\mathcal{M}_{g}$. We use the WP metric as the bundle metric and we put the Ricci metric on the base variety $\mathcal{M}_{g}$. We do this because of the technique difficulty that the WP metric is incomplete and thus there is trouble in defining the adjoint operator $\bar{\partial}^{*}$. After setting up the section spaces appropriately, the proof of this theorem is a direct application of the goodness of these metrics and can be found in [8].

Now we combine the above result with the dual Nakano negativity of the Weil-Petersson metric. In [8] we proved the following Nakano-type vanishing theorem

Theorem 5.2. The $L^{2}$ cohomology group vanish:

$$
H_{(2)}^{0, q}\left(\left(\mathcal{M}_{g}, \omega_{\tau}\right),\left(T_{\mathcal{M}_{g}}, \omega_{W P}\right)\right)=0
$$

unless $q=3 g-3$.

The proof is similar to proof of Nakano vanishing theorem in the case when the base manifold is closed. It depends on the Kodaira-Nakano identity

$$
\square_{\bar{\partial}}=\square_{\nabla}+\sqrt{-1}\left[\nabla^{2}, \Lambda\right]
$$

We then apply the dual Nakano negativity of the WP metric to get the vanishing theorem by using the goodness to deal with integration by part to show that there is no boundary term. We note here that, in the proof of Nakano vanishing theorem, we only need the negativity of the curvature of the bundle metric.

The above two theorems imply a result of Hacking [4]

Corollary 5.1. The pair $\left(\overline{\mathcal{M}}_{g}, D\right)$ is infinitesimally rigid.

\section{§. Goodness of the Ricci and Perturbed Ricci Metrics}

In this last section we discuss the goodness of the metrics on $T_{\mathcal{M}_{g}}^{*}(\log D)$ induced by the Ricci and perturbed Ricci metrics. These results here are more difficult than that in Section 3, the goodness of the Weil-Petersson metric. Our main theorem is

Theorem 6.1. The metrics $\tau^{*}$ and $\widetilde{\tau}^{*}$ on $T_{\mathcal{M}_{g}}^{*}(\log D)$ induced by the Ricci and perturbed Ricci metrics are good in the sense of Mumford. 
By Lemma 3.1, we need to show the estimates (3.9), (3.10), (3.12) and (3.14) hold when the metric $g$ is the Ricci metric $\tau$ or the perturbed Ricci metric $\widetilde{\tau}$.

Here we only describe the proof of the estimates of the goodness of the Ricci metric $\tau$. Please see [8] for the perturbed Ricci metric.

In this case, the zero-th order estimate follows from Theorem 2.3 directly.

Now we consider the first order estimate. We let $\widetilde{\Gamma}_{i j}^{k}$ be the Christoffell symbol of the Ricci metric under the pinching coordinates. To show the first order estimate, we need to check that $\widetilde{\Gamma}_{i j}^{k}$ satisfy the inequalities (3.9) and (3.10). By Theorem 3.2 of $[6]$ we have

$$
\widetilde{\Gamma}_{k p}^{i}=\tau^{i \bar{j}} h^{\alpha \bar{\beta}}\left(\sigma_{1} \int_{X} \xi_{p}\left(e_{k \bar{j}}\right) e_{\alpha \bar{\beta}} d v\right)+\Gamma_{k p}^{i}
$$

where $\Gamma_{k p}^{i}$ is the Christoffell symbol of the WP metric. In order to prove the first order estimate for the Ricci metric, by Remark 3.1 and Lemma 3.8, we only need to estimate the tensor part. Thus the first order estimate is equivalent to

Lemma 6.1. For any $i, k$ and $p$, we have

$$
\left|\tau^{i \bar{j}} h^{\alpha \bar{\beta}}\left(\sigma_{1} \int_{X} \xi_{p}\left(e_{k \bar{j}}\right) e_{\alpha \bar{\beta}} d v\right)\right|=O\left(\Lambda_{p}\right) .
$$

The central part of the proof of this lemma is following estimates which is the optimal version of the results in $[6]$ :

\section{Lemma 6.2.}

$$
\left|P\left(\widetilde{e}_{i \bar{j}}\right)\right|_{L^{1}}= \begin{cases}O\left(\frac{u_{i}^{3}}{\left|t_{i}\right|^{2}}\right) & i=j \leq m \\ O\left(\frac{u_{i}^{3} u_{j}^{3}}{\left|t_{i} t_{j}\right|}\right) & i, j \leq m, i \neq j \\ O\left(\frac{u_{i}^{3}}{\left|t_{i}\right|}\right) & i \leq m<j \\ O\left(\frac{u_{j}^{3}}{\left|t_{j}\right|}\right) & j \leq m<i \\ O(1) & i, j>m .\end{cases}
$$

Similarly, the second order estimate follows from sharpening the estimates in [6]. Please see [8] for details.

It is well known that the line bundle $K_{\overline{\mathcal{M}}_{g}}+[D]$, which is the determinant bundle of $T_{\mathcal{M}_{g}}^{*}(\log D)$, is positive. In [7] we proved

Theorem 6.2. The logarithmic cotangent bundle $\bar{E}=T_{\overline{\mathcal{M}}_{g}}^{*}(\log D)$ is stable with respect to its first Chern class. 
Since $\bar{E}$ is stable, by the works of Donaldson-Uhlenbeck-Yau [3], [17] there is a Hermitian-Einstein metric on $\bar{E}$ with respect to any metric in the class $c_{1}(\bar{E})$ which gives an orbifold Chern number inequality. However, the metric induced by the Kähler-Einstein metric, if it is good, will give a stronger Chern number inequality. Thus a more interesting question is whether the metric on $T_{\mathcal{M}_{g}}^{*}(\log D)$ induced by the Kähler-Einstein metric on $\mathcal{M}_{g}$ is good or not. The difficulty rises from that it is very hard to control the off-diagonal terms in the local expression of the Kähler-Einstein metric with respect to the pinching coordinates. We believe that this difficulty can be solved by studying the Kähler-Ricci flow on $\mathcal{M}_{g}$ with the Ricci metric as the initial metric and showing that the goodness is preserved under the flow.

\section{References}

[1] L. Ahlfors and L. Bers, Riemann's mapping theorem for variable metrics, Ann. of Math. (2) 72 (1960), 385-404.

[2] P. Deligne and D. Mumford, The irreducibility of the space of curves of given genus, Inst. Hautes Études Sci. Publ. Math. No. 36 (1969), 75-109.

[3] S. K. Donaldson, Anti self-dual Yang-Mills connections over complex algebraic surfaces and stable vector bundles, Proc. London Math. Soc. (3) 50 (1985), no. 1, 1-26.

[4] P. Hacking, The moduli space of curves is rigid, arXiv:math.AG/0509567, 2006.

[5] L. Keen, Collars on Riemann surfaces, in Discontinuous groups and Riemann surfaces (Proc. Conf., Univ. Maryland, College Park, Md., 1973), 263-268. Ann. of Math. Studies, 79, Princeton Univ. Press, Princeton, N.J.

[6] K. Liu, X. Sun and S.-T. Yau, Canonical metrics on the moduli space of Riemann surfaces. I, J. Differential Geom. 68 (2004), no. 3, 571-637.

[7] Canonical metrics on the moduli space of Riemann surfaces. II, J. Differential Geom. 69 (2005), no. 1, 163-216.

[8] _ Good metrics on the moduli space of Riemann surface I, preprint, 2006.

[9] _ Good metrics on the moduli space of Riemann surface II, preprint, 2006.

[10] H. Masur, Extension of the Weil-Petersson metric to the boundary of Teichmuller space, Duke Math. J. 43 (1976), no. 3, 623-635.

[11] D. Mumford, Hirzebruch's proportionality theorem in the noncompact case, Invent. Math. 42 (1977), 239-272.

[12] L. Saper, $L^{2}$-cohomology of the Weil-Petersson metric, in Mapping class groups and moduli spaces of Riemann surfaces (Göttingen, 1991/Seattle, WA, 1991), 345-360, Contemp. Math., 150, Amer. Math. Soc., Providence, RI.

[13] G. Schumacher, Harmonic maps of the moduli space of compact Riemann surfaces, Math. Ann. 275 (1986), no. 3, 455-466.

[14] _ The curvature of the Petersson-Weil metric on the moduli space of KählerEinstein manifolds, in Complex analysis and geometry, 339-354, Plenum, New York.

[15] Y. T. Siu, Curvature of the Weil-Petersson metric in the moduli space of compact KählerEinstein manifolds of negative first Chern class, in Contributions to several complex variables, 261-298, Vieweg, Braunschweig.

[16] S. Trapani, On the determinant of the bundle of meromorphic quadratic differentials on the Deligne-Mumford compactification of the moduli space of Riemann surfaces, Math. Ann. 293 (1992), no. 4, 681-705. 
[17] K. Uhlenbeck and S.-T. Yau, On the existence of Hermitian-Yang-Mills connections in stable vector bundles, Comm. Pure Appl. Math. 39 (1986), no. S, suppl., S257-S293.

[18] S. A. Wolpert, Chern forms and the Riemann tensor for the moduli space of curves, Invent. Math. 85 (1986), no. 1, 119-145.

[19] _ Asymptotics of the spectrum and the Selberg zeta function on the space of Riemann surfaces, Comm. Math. Phys. 112 (1987), no. 2, 283-315.

[20] - The hyperbolic metric and the geometry of the universal curve, J. Differential Geom. 31 (1990), no. 2, 417-472. 